\title{
Effect of different long-chain fatty acids on cholecystokinin release in vitro and energy intake in free-living healthy males
}

\author{
Charlotte J. Harden ${ }^{1,2,3}$, Adam N. Jones ${ }^{1}$, Tannia Maya-Jimenez ${ }^{1}$, Margo E. Barker ${ }^{1}$, Natalie J. Hepburn ${ }^{4}$, \\ Iveta Garaiova ${ }^{4}$, Sue F. Plummer ${ }^{4}$ and Bernard M. Corfe ${ }^{1 *}$ \\ ${ }^{1}$ Academic Unit of Surgical Oncology, Department of Oncology, School of Medicine and Biomedical Sciences, \\ University of Sheffield, Beech Hill Road, Sheffield S1O 2RX, UK \\ ${ }^{2}$ Cultech Limited, Unit 3, Christchurch Road, Baglan Industrial Park, Port Talbot, West Glamorgan SA12 $7 B Z$, UK \\ ${ }^{3}$ Centre for Food Innovation, Stoddart Building, Sheffield Hallam University, Howard Street, Sheffield S1 1 WB, UK \\ ${ }^{4}$ Obsidian Research Limited, Unit 2, Christchurch Road, Baglan Industrial Park, Port Talbot, West Glamorgan SA12 7 BZ, UK
}

(Submitted 24 February 2011 - Final revision received 5 October 2011 - Accepted 8 October 2011 - First published online 7 February 2012)

\section{Abstract}

Long-chain fatty acids have been shown to suppress appetite and reduce energy intake (EI) by stimulating the release of gastrointestinal hormones such as cholecystokinin (CCK). The effect of NEFA acyl chain length on these parameters is not comprehensively understood. An in vitro screen tested the capacity of individual NEFA (C12 to C22) to trigger CCK release. There was a gradient in CCK release with increasing chain length. DHA (C22) stimulated significantly $(P<0.01)$ more CCK release than all other NEFA tested. Subsequently, we conducted a randomised, controlled, crossover intervention study using healthy males ( $n$ 18). The effects of no treatment (NT) and oral doses of emulsified DHA-rich (DHA) and oleic acid (OA)-rich oils were compared using $24 \mathrm{~h}$ EI as the primary endpoint. Participants reported significantly $(P=0.039)$ lower total daily EI (29\% reduction) with DHA compared to NT. There were no differences between DHA compared to OA and OA compared to NT. There was no between-treatment difference in the time to, or EI of, the first post-intervention eating occasion. It is concluded that NEFA stimulate CCK release in a chain length-dependent manner up to C22. These effects may be extended to the in vivo setting, as a DHA-based emulsion significantly reduced short-term EI.

Key words: Secretin tumour cells-1: Long-chain fatty acids: Cholecystokinin: Energy intake

Food ingestion stimulates satiety and inhibits energy intake (EI) via complex mechanisms, in which signals arising from the gastrointestinal tract play a critical role ${ }^{(1)}$. The presence of dietary fat in the gastrointestinal tract, for example, has been shown to trigger the endogenous release of many anorectic hormones including cholecystokinin $(\mathrm{CCK})^{(1,2)}$.

CCK is secreted from I-cells in the duodenum ${ }^{(3)}$. Dietary fats are hydrolysed from TAG into monoacylglycerols and NEFA by lingual, gastric and pancreatic lipase before absorption. Luminal NEFA (rather than TAG) trigger CCK release and longer-chain, unsaturated NEFA appear to be the most potent stimulants ${ }^{(4-7)}$. Matzinger et al. ${ }^{(8)}$ suggest that the generation of long-chain NEFA via lipid hydrolysis is a critical step in fat-induced inhibition of EI and that this signal is mediated via CCK release and CCK-A receptors ${ }^{(8)}$. Basal human plasma CCK concentrations are generally in the low picomolar (approximately $1 \mathrm{pm}$ ) range but can increase 10-fold following high-fat meals ${ }^{(9)}$. It is hypothesised that CCK regulates appetite by inhibiting gastric emptying and promoting gastric mechanoreceptor stimulation ${ }^{(9)}$.

The secretin tumour cell (STC)-1 enteroendocrine cell line provides a model to study intestinal hormone expression since it demonstrates some features of native intestinal hormone-secreting cells. It has been used to show acyl chain length-dependent NEFA-induced CCK release up to $\mathrm{C} 18^{(5,10)}$. In the present investigation, we tested the effects of NEFA, with longer chain lengths than previously reported, on CCK release. The NEFA most effective at stimulating CCK in vitro was incorporated into an oil emulsion to test its effects on in vivo $24 \mathrm{~h} \mathrm{EI}$.

\section{Experimental methods}

\section{Cholecystokinin release cell assay}

Reagents were from Sigma (Poole, UK) unless otherwise stated. STC-1 cells were kindly supplied by Dr Rumio,

Abbreviations: CCK, cholecystokinin; EI, energy intake; NT, no treatment; OA, oleic acid; STC, secretin tumour cell.

*Corresponding author: Dr B. M. Corfe, fax +44114271 3314, email b.m.corfe@sheffield.ac.uk 
University of Milan. Cells were maintained as previously described $^{(11)}$ and seeded into twelve-well plates, at $6 \times 10^{5}$ per well, $48 \mathrm{~h}$ before incubation with solutions of dodecanoic (12:0), cis-9-octadecenoic (18:1), all cis-9,12-octadecadienoic (18:2), cis-9, trans-11-octadecadienoic (18:2), cis-5,9,12-octadecatrienoic (18:3), all cis-5,8,11,14,17-eicosapentaenoic (20:5) and all cis-4,7,10,13,16,19-docosahexaenoic (22:6) at final concentrations of $66 \mu \mathrm{m}$, for $60 \mathrm{~min}$. Supernatant levels of CCK were assayed by ELISA according to the manufacturer's protocol (Phoenix Europe GmbH, Karlsruhe, Germany). CCK concentrations were normalised against total cellular protein by quantifying the cell lysates according to the manufacturer's protocol (Thermo Scientific, Rockford, IL, USA).

\section{Prospective food intake in response to consumption of long-chain fatty acids-based emulsions}

Subjects. A total of eighteen subjects were recruited via email and poster advertisements around the University of Sheffield. Exclusion criteria included: BMI outside normal and overweight UK categories $\left(<20\right.$ and $\left.>29.9 \mathrm{~kg} / \mathrm{m}^{2}\right)$, recent food poisoning, diagnosed gastrointestinal, metabolic or endocrine disorders, food allergies, use of nutritional supplements, smoking, age $<18$ and $>65$ years and having implantable electronic devices (contraindicated for bioelectrical impedance analysis). Height and weight were recorded to the nearest $0 \cdot 1 \mathrm{~cm}$ and $0 \cdot 1 \mathrm{~kg}$, respectively, to calculate BMI to the nearest $0 \cdot 1 \mathrm{~kg} / \mathrm{m}^{2}$. Bioelectrical impedance analysis was undertaken using BodyStat 1500 (BodyStat Limited, Isle of Man, British Isles). Body fat and lean weight were recorded to the nearest $0 \cdot 1 \%$ or $0 \cdot 1 \mathrm{~kg}$, respectively.

Study design. This was a controlled, single-blind, threeway crossover study designed to examine the effects of two long-chain fatty acid emulsions, compared to no treatment (NT), on prospective food intake. The study was conducted according to the guidelines laid down in the Declaration of Helsinki and all procedures involving human subjects were approved by the University of Sheffield; (SMBRER158). Written informed consent was obtained from all subjects. Treatments were orally administered, low-energy (145 kJ (35 kcal)), 45\% oil-in-water emulsions masked with lemon flavouring, comprising either predominantly DHA (DHA) or predominantly oleic acid (OA). These were offered in randomised order as a single $6 \mathrm{ml}$ dose.

Interventions took place on three different occasions, separated by a week. The day before each visit, subjects consumed a fixed-load evening meal after which they fasted (excluding water) for $12 \mathrm{~h}$. To ascertain glycaemia (a measure of fasting compliance), a blood sample was applied to an OneTouchR UltraR Test Strip with FastDrawTM design (reference range: 1.1-33.3 mmol/1; Lifescan, Inc., Bucks, UK). Emulsions were administered to ensure $100 \%$ compliance. Then, 20 min after taking the emulsion, subjects consumed a fixed-load breakfast, and then were free to continue normal activities. The following day, a researcher (who was blinded to treatment allocation) telephoned subjects to collect food intake data using the automated multiple-pass $24 \mathrm{~h}$ recall method $^{(12)}$. Dietary information was analysed using NetWISP software (version 3.0 for Windows; Tinuviel Software, Warrington, UK). An eating occasion was defined as an event providing $\geq 210 \mathrm{~kJ}$ ( $50 \mathrm{kcal})^{(13)}$. Overall, fifteen subjects completed the study according to protocol. No adverse effects related to the study products were reported.

\section{Statistical methods}

One-way ANOVA using PASW Statistics (version 18.0 for Windows, SPSS, Inc., Chicago, IL, USA) was used for initial analyses. Post hoc comparisons were analysed using the Tukey honestly significant difference test. Data are presented as mean values and standard deviations.

\section{Results}

\section{Effects of NEFA on in vitro cholecystokinin release}

The NEFA elicited significantly $(P<0.01)$ different degrees of CCK release both using absolute quantification and when normalised for biomass. The order of NEFA-induced CCK production was DHA (22:6), EPA 20:5), pinolenic acid (18:3), linoleic acid (18:2), oleic acid (18:1), conjugated linoleic acid (18:2) and lauric acid (12:0). The ethanol control assayed $1 \cdot 16 \mathrm{ng} / \mu \mathrm{g}$ (Fig. 1). Post hoc comparisons indicated that DHA was significantly $(P<0.01)$ more effective at stimulating CCK release in STC-1 cells than any other NEFA tested.

\section{Effect of DHA, oleic acid and no treatment on prospective food intake and time to first eating occasion}

All glycaemic measurements were within the normal fasting range $^{(14)}$. There was no difference between treatments in time to first post-intervention eating occasion or the energy, protein, carbohydrate and fat contents of these meals. There was a significant $(P=0.048)$ difference in total daily EI across treatments. Post hoc comparisons indicated that subjects reported significantly $(P=0.039)$ lower EI with DHA relative to NT. There was no difference in total daily EI for DHA compared to OA or OA compared to NT (Table 1). Although reduction in the intake of all macronutrients accounted for the fall in EI, treatment did not affect the intake of any individual macronutrient.

\section{Discussion}

This study shows that DHA increases the in vitro release of CCK and decreases EI in healthy men. Several studies show that NEFA-induced CCK secretion in STC-1 cells is directly proportional to chain length, but only NEFA up to C18 have been examined ${ }^{(5,10,15)}$. Our in vitro results extend the literature by demonstrating that this chain length relationship exists from C12 up to at least C22. Our data also show that CCK secretion, triggered by C18 NEFA, differed by degree of unsaturation. These preliminary findings may indicate that the NEFAmediated CCK-response may be sensitive to both desaturation and double-bond configuration. 


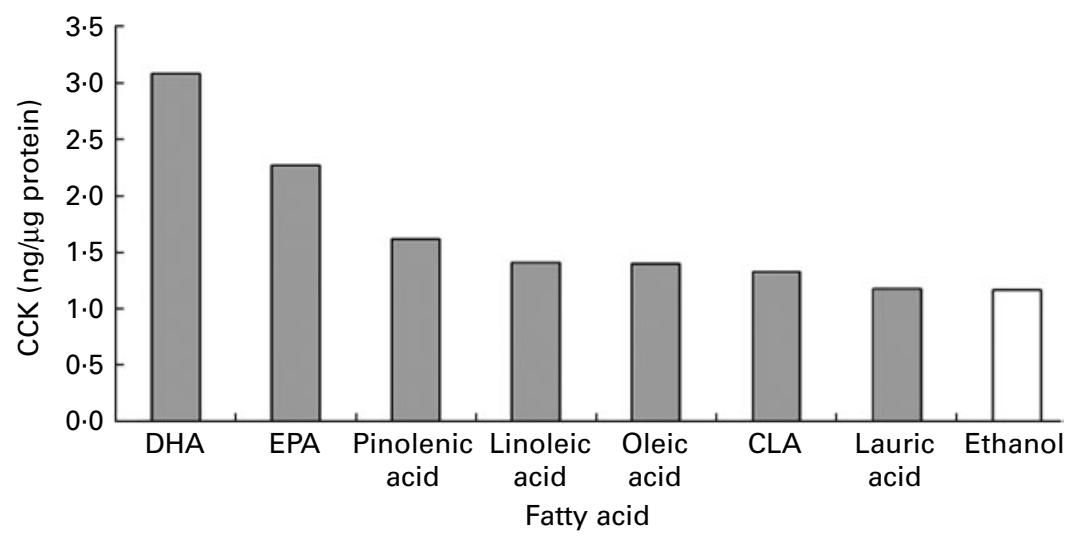

Fig. 1. Cholecystokinin (CCK) release from secretin tumour cells (STC)-1 in response to NEFA; ng CCK release from STC-1 cells per $\mu$ g protein. Values are means and standard deviations of three experiments (SD were $<2 \%$ of the mean). All NEFA produced significantly $(P<0.01)$ more CCK than the ethanol control except lauric acid. Post hoc comparisons using the Tukey honestly significant difference test indicate that all NEFA stimulated significantly $(P<0.01)$ different quantities of CCK production compared to each other. CLA, conjugated linoleic acid.

DHA was the most effective NEFA at stimulating CCK release and this led to an assessment of its effects on prospective food intake in fifteen healthy men. In this study, DHA significantly reduced EI by $3558 \mathrm{~kJ}$ compared to NT. If this level of EI reduction was sustained in the longer term, it could lead to weight loss. A study testing such effects in overweight subjects is warranted. OA yielded a non-significant reduction in EI compared to NT. The EI reduction must be attributable to a specific physiological response as DHA and OA were isoenergetic. Other researchers suggest that the effect of long-chain NEFA on appetite is partly mediated by increased CCK production ${ }^{(16,17)}$. STC-1 cells and EE cells in vivo are, however, stimulated to release a cascade of appetitesuppressing hormones in response to lipids ${ }^{(17-19)}$. It cannot therefore be assumed that the EI reduction observed here was mediated solely by CCK.

DHA did not affect the first post-treatment eating occasion; EI was suppressed throughout the day. This suggests that the time course by which fatty acids affect food consumption could extend up to $24 \mathrm{~h}$. Food ingestion stimulates the release of numerous hormones into the blood, some of which have a direct effect on gastric emptying and others have longerlasting postprandial effects on satiety ${ }^{(18)}$. Other studies have reported greater fullness and reduced hunger after consuming high compared to low DHA/EPA diets relative to olive oil ${ }^{(20)}$ and reduced EI up to $48 \mathrm{~h}$ after consumption of high-polyunsaturated compared to high-MUFA meals ${ }^{(21)}$.

Comparing the effects of equienergetic oils eliminates the possibility that EI alteration is mediated by energy and/or total lipid ingestion, and suggests that the effects are due to differences in the oils' fatty acid composition. OA was demonstrated to have some bioactivity in the in vitro experiment and this was reflected in vivo. Other studies using oil containing predominantly OA have also observed effects suggesting that it is not biologically inert ${ }^{(22,23)}$. Evidence suggests that emulsification of fish oils improves digestion and absorption

Table 1. Fasting glycaemia, time to first eating occasion, energy, protein, carbohydrate and fat intakes for the first post-intervention eating occasion $(\geq 210 \mathrm{~kJ})$ and total study day (post-intervention to 23.59 hours) by treatment (Mean values and standard deviations)

\begin{tabular}{|c|c|c|c|c|c|c|c|c|}
\hline \multirow[b]{3}{*}{ Parameter } & \multicolumn{6}{|c|}{ Treatment } & & \\
\hline & \multicolumn{2}{|c|}{ NT } & \multicolumn{2}{|c|}{ OA } & \multicolumn{2}{|c|}{$\mathrm{DHA}$} & \multicolumn{2}{|c|}{ Statistical significance } \\
\hline & Mean & SD & Mean & SD & Mean & SD & $F$ & $P$ \\
\hline Fasting glycaemia (mmol/l) & 5.4 & 0.36 & $5 \cdot 3$ & 0.59 & $5 \cdot 5$ & 0.47 & 0.474 & 0.626 \\
\hline Time to first eating occasion (min) & 96 & 64 & 133 & 99 & 121 & 64 & 0.908 & 0.411 \\
\hline \multicolumn{9}{|l|}{ First eating occasion } \\
\hline Energy (kJ) & 3148 & 1984 & 3035 & 1733 & 2507 & 1302 & 0.616 & 0.545 \\
\hline Protein $(\mathrm{g})$ & 34 & 23 & 29 & 18 & 26 & 15 & 0.621 & 0.542 \\
\hline Carbohydrate (g) & 88 & 59 & 86 & 53 & 78 & 49 & 0.124 & 0.884 \\
\hline Fat $(g)$ & 32 & 23 & 32 & 21 & 22 & 18 & $1 \cdot 109$ & 0.339 \\
\hline \multicolumn{9}{|l|}{ Total study day } \\
\hline Energy (kJ) & $12097^{\star}$ & 5429 & 9912 & 2733 & 8539 & 2720 & $3 \cdot 262$ & $0.048 \dagger$ \\
\hline Protein $(\mathrm{g})$ & 111 & 36 & 102 & 39 & 87 & 37 & 1.564 & 0.221 \\
\hline Carbohydrate (g) & 314 & 129 & 273 & 79 & 259 & 87 & $1 \cdot 219$ & 0.306 \\
\hline Fat $(\mathrm{g})$ & 110 & 48 & 102 & 37 & 77 & 27 & 2.935 & 0.064 \\
\hline
\end{tabular}

NT, no treatment; OA, oleic acid.

* Mean value was significantly different from that of DHA ( $P=0.039$, post hoc comparisons using the Tukey honestly significant difference test).

†There was significant difference in total daily El across treatments $(P=0.039)$. 
of $n-3$ long-chain polyunsaturated NEFA due to solubility modification ${ }^{(24)}$. Using our novel oil-emulsion approach may offer greater study standardisation and limit between study variance caused by differential meal response.

Overall, our results show that NEFA can increase appetite hormone CCK release in vitro and this effect is chain-lengthdependent. Compared to NT, $24 \mathrm{~h}$ EI was reduced after consumption of a predominantly DHA (22:5) oil-emulsion, but not after a predominantly OA (18:0) oil-emulsion. Further studies to examine if reduced EI is sustained and leads to weight loss are warranted, as are evaluations in female and obese subjects.

\section{Acknowledgements}

The present study was jointly funded by BBSRC and the Welsh Assembly Government via a Knowledge Transfer Award. S. F. P., N. J. H. and I. G. work for Obsidian Research Limited, who are co-recipients of a Knowledge Transfer Award. The contributions of the authors were as follows: C. J. H. conducted the cell biology studies and dietary analysis aspect of the human intervention work, and undertook all analyses of data as presented. A. N. J. and T. M.-J. conducted the human intervention work and undertook first-pass analysis of the data. M. E. B. oversaw the analysis of the dietary data and the development of the manuscript. N. J. H. conceived, developed and supervised the in vitro aspects of the work. I. G. oversaw the statistical analysis of the data. S. F. P. co-conceived and COsupervised the study and the development of the manuscript. B. M. C. conceived and supervised the study, took responsibility for the manuscript and edited its successive versions.

\section{References}

1. Cummings DE \& Overduin J (2007) Gastrointestinal regulation of food intake. J Clin Invest 117, 13-23.

2. Seimon RV, Wooster TM, Otto B, et al. (2009) The droplet size of intraduodenal fat emulsions influences antropyloroduodenal motility, hormone release, and appetite in healthy males. Am J Clin Nutr 89, 1729-1736.

3. Lay JM, Bane G, Brunkan CS, et al. (2004) Enteroendocrine cell expression of a cholecystokinin gene construct in transgenic mice and cultured cells. Am J Physiol Gastrointest Liver Physiol 288, 354-361.

4. Little TJ, Russo A, Meyer JH, et al. (2007) Free fatty acids have more potent effects on gastric emptying, gut hormones and appetite than triglycerides. Gastroenterology 133, $1124-1131$.

5. McLaughlin J, Grazia Lucà M, Jones MN, et al. (1999) Fatty acid chain length determines cholecystokinin secretion and effect on human gastric motility. Gastroenterology 116, $46-53$.

6. Costarelli V \& Sanders TAB (2001) Acute effects of dietary fat composition on postprandial plasma bile acid and cholecystokinin concentrations in healthy premenopausal women. Br J Nutr 86, 471-477.

7. Jonkers IJ, Ledeboer M, Steens J, et al. (2000) Effects of very long chain versus long chain triglycerides on gastrointestinal motility and hormone release in humans. Dig Dis Sci $\mathbf{4 5}$, $1719-1726$.

8. Matzinger D, Degen L, Drewe J, et al. (2000) The role of long chain fatty acids in regulating food intake and cholecystokinin release in humans. Gut 46, 688-693.

9. Feinle-Bisset C, Horowitz M \& Little TJ (2005) Role of cholecystokinin in appetite control and body weight regulation. Int J Obes 6, 297-306.

10. Sidhu SS, Thompson DG, Warhurst G, et al. (2000) Fatty acid-induced cholecystokinin secretion and changes in intracellular $\mathrm{Ca}^{2+}$ in two enteroendocrine cell lines, STC-1 and GLUTag. J Physiol 528, 165-176.

11. Leech SH, Evans CA, Shaw L, et al. (2008) Proteomic analysis of intermediate filaments reveals cytokeratin8 is highly accelerated-implications for colorectal epithelial homeostasis. Proteomics 8, 279-288.

12. Blanton CA, Moshfegh AJ, Baer DJ, et al. (2006) The USDA Automated Multiple-Pass Method accurately estimates group total energy and nutrient intake. J Nutr 136, 2594-2599.

13. Gibney MJ \& Wolever TMS (1997) Periodicity of eating and human health: present perspective and future directions. Br J Nutr 77, 3-5.

14. National Health Service (2011) Monitoring Blood Glucose Levels. National Health Service. http://www.nhs.uk/Conditions/ Diabetes-type1/Pages/Glucoselevels.aspx

15. Tanaka T, Katsuma S, Adachi T, et al. (2008) Free fatty acids induce cholesystokinin secretion through GPR120. Naunyn Schmiedebergs Arch Pharmacol 377, 523-527.

16. Hughes G, Boylans E, Williams N, et al. (2008) The effect of Korean pine nut oil (PinnoThin ${ }^{\mathrm{TM}}$ ) on food intake, feeding behaviour and appetite: a double-blind placebo-controlled trial. Lipids Health Dis 7, 6-20.

17. Feinle C, Rades T, Otto B, et al. (2001) Fat digestion modulates gastrointestinal sensations induced by gastric distension and duodenal lipid in humans. Gastroenterology 120, $1100-1107$.

18. de Graff C, Blom WAM, Smeets PAM, et al. (2004) Biomarkers of satiation and satiety. J Am Clin Nutr 79, 946-961.

19. Geraedts MC, Troost FJ \& Saris WH (2009) Peptide-YY is released by the intestinal cell line STC-1. J Food Sci $\mathbf{7 4}$, 79-82.

20. Parra D, Ramel A, Bandarra N, et al. (2008) A diet rich in long chain omega-3 fatty acids modulates satiety in overweight and obese volunteers during weight loss. Appetite 51, 676-680.

21. Lawton CL, Delargy HJ, Brockman J, et al. (2000) The degree of saturation of fatty acids influences post-ingestive satiety. Br J Nutr 83, 473-482.

22. Pasman WJ, Heimerikx J, Rubingh CM, et al. (2008) The effect of Korean pine nut oil on in vitro CCK release, on appetite sensations and on gut hormones in post-menopausal overweight women. Lipids Health Dis 7, 10.

23. Fontani G, Corradeschi F, Felici A, et al. (2005) Blood profiles, body fat and mood state in healthy subjects on different diets supplemented with omega-3 polyunsaturated fatty acids. Eur J Clin Invest 35, 499-507.

24. Garaiova I, Guschina IA, Plummer SF, et al. (2007) A randomised cross-over trial in healthy adults indicating improved absorption of omega- 3 fatty acids by pre-emulsification. Nutr J 6, 70-74. 